\title{
As Relações entre Depressão Materna e Relatos Maternos acerca do Envolvimento Paterno: Um Estudo Longitudinal
}

\author{
Patrícia Alvarenga ${ }^{1}$ \\ João Marcos de Oliveira \\ Quele de Souza Gomes \\ Luna Maiana Araújo Freitas \\ Programa de Pós-Graduação em Psicologia da Universidade Federal da Bahia, \\ Salvador, BA, Brasil
}

\begin{abstract}
Resumo
Este estudo longitudinal avaliou as relações entre indicadores de depressão da mãe e sua percepção acerca do envolvimento paterno. Participaram 19 mães de meninos, recrutadas em maternidades públicas da cidade de Salvador. A depressão materna foi avaliada no primeiro e no $18^{\circ}$ mês da criança (Inventário de Depressão de Beck - BDI) e no $30^{\circ}$ mês foi realizada uma entrevista estruturada sobre a percepção da mãe acerca do envolvimento paterno. Os escores de depressão materna no primeiro mês de vida do bebê estiveram positivamente correlacionados à percepção da mãe acerca da disponibilidade paterna aos 30 meses, particularmente, em relação aos cuidados de rotina, lazer e saúde da criança. Discute-se o impacto da depressão pós-parto sobre esta dimensão específica do envolvimento paterno, enfatizando as mudanças que podem ocorrer na dinâmica das interações familiares no período da transição para a parentalidade. Sugere-se a realização de novos estudos sobre depressão materna e envolvimento paterno, tendo como informantes tanto os pais quanto as mães.
\end{abstract}

Palavras-chave: Depressão materna, envolvimento paterno, infância.

\section{The Relations between Maternal Depression and Maternal Reports about Father Involvement: A Longitudinal Study}

\begin{abstract}
This longitudinal study evaluated the relationship between indicators of mother's depression and their perception of paternal involvement. Nineteen mothers of boys, recruited at public maternity hospitals in Salvador, Bahia, were assessed for maternal depression with the Beck's Depression Inventory (BDI) at the first and $18^{\text {th }}$ month of the child. The participants responded a structured interview regarding maternal perceptions about paternal involvement at the $30^{\text {th }}$ month of the child. The maternal depression scores at the first month of the baby's life were positively correlated to maternal perceptions of paternal availability at the $30^{\text {th }}$ month, particularly concerning to routine care, leisure and child health. The impact of postpartum depression on this specific dimension of paternal involvement are discussed, emphasizing the changes that may occur in the dynamics of family interactions during the transition
\end{abstract}

Endereço para correspondência: Rua Aristides Novis, No 197, Salvador, BA, Brasil 40210-730. Fone: (71) 3283-6437. E-mail: palvarenga66@gmail.com

Financiamento: Fundação de Amparo à Pesquisa do Estado da Bahia (FAPESB). 
to parenthood. Further studies are suggested concerning the relation between maternal depression and paternal involvement, with reports of both fathers and mothers.

Keywords: Maternal depression, paternal involvement, childhood.

\section{Las Relaciones entre la Depresión Materna y los Informes Maternos sobre el Envolvimiento Paterno: Un Estudio Longitudinal}

\section{Resumen}

Este estudio evaluó la relación entre los indicadores de depresión materna y la percepción de la madre respecto al envolvimiento paterno. Participaron 19 madres de niños varones. La depresión materna fue evaluada en el primero y en el $18^{\circ}$ mes del niño (Inventário de Depressão de Beck- BDI). En el trigésimo mes, se realizó una entrevista estructurada sobre la percepción de la madre sobre el envolvimiento paterno. Los escores de depresión materna en el primer mes de vida del bebé se correlacionaron positivamente con la percepción de la madre respecto a la disponibilidad paterna a los 30 meses, en particular, la disponibilidad para los cuidados básicos y la salud, y para jugar con el niño. Se discute el impacto de la depresión posparto en esta dimensión particular del envolvimiento paterno, con énfasis en los cambios que pueden ocurrir en la dinámica de las interacciones familiares durante la transición a la parentalidad. Se sugieren más estudios sobre la relación entre la depresión materna y la participación de los padres, con los informes de los padres y de las madres.

Palabras clave: Depresión materna, envolvimiento paterno, infância.

A depressão foi identificada como uma das principais causas de incapacidade na população mundial e tende a acometer aproximadamente de 1,5 a 3 vezes mais mulheres do que homens (Associação Americana de Psiquiatria [APA], 2013). Dados recentes ainda indicam que populações de jovens entre 18 e 29 anos de idade têm 3 vezes mais chances de apresentar transtornos depressivos maiores, do que os indivíduos com 60 anos de idade ou mais (APA, 2013) e apontam para a alta prevalência de depressão pós-parto, com índices entre 33 e 38\% (Cruz, Simões, \& Faisal-Cury, 2005; Gress-Smith, Luecken, Lemery-Chalfant, \& Howe, 2012). Nesse sentido, a depressão tende a afetar particularmente a população feminina e pode manifestar-se inicialmente durante o processo de transição para a maternidade, repercutindo de modo significativo nas relações familiares.

A ocorrência da depressão após a gestação é designada na literatura através de duas denominações: depressão pós-parto e depressão materna. Contudo, não existe uma diferenciação entre os dois termos, no que diz respeito à sintoma- tologia ou caracterização dos quadros. Os dois tipos de depressão distinguem-se em virtude do momento no qual os sintomas emergem (Silva \& Piccinini, 2009). O termo depressão pós-parto é utilizado quando se trata de um quadro detectado no período entre quatro (APA, 2013) e seis semanas após o parto (Organização Mundial da Saúde [OMS], 1997), enquanto o termo depressão materna, costuma ser empregado para referir-se a sintomas depressivos que acometem a mãe em qualquer fase do desenvolvimento da criança, excluindo-se o período logo após o parto (Frizzo \& Piccinini, 2007).

Ambos os quadros apresentam os mesmos marcadores típicos de outros transtornos depressivos, como humor deprimido, desânimo, baixa capacidade de concentração e distúrbios de sono e apetite (APA, 2013). No que se refere à repercussão da depressão da mãe sobre a interação com a criança, a literatura aponta de forma consistente para manifestações mais frequentes de afeto negativo, intrusividade, apatia e, de modo mais geral, para a baixa responsividade em relação aos sinais e comportamentos da criança 
(Alvarenga \& Palma, 2013; Field, 1995, 2010; Lovejoy, Graczyk, O’Hare, \& Neuman, 2000).

A depressão materna, seja no período pós-parto, seja em etapas posteriores do desenvolvimento dos filhos, tende a produzir impacto negativo sobre a interação familiar, a relação conjugal e, principalmente, sobre o desenvolvimento socioemocional da criança. Os filhos de mães deprimidas tendem a apresentar mais problemas de comportamento (Boyle \& Pickles, 1997; Loosli \& Loureiro, 2010; Mian, Tango, Lopes, \& Loureiro, 2009), reatividade emocional (Alvarenga, Oliveira, \& Lins, 2012) e episódios depressivos (Pizeta, Silva, Cartafina, \& Loureiro, 2013). Além disso, quando comparados aos filhos de mães não deprimidas, aqueles que têm mães deprimidas tendem a ser mais ansiosos e a apresentar menor responsividade, atenção, sorrisos e interação corporal nas relações interpessoais (Silva \& Piccinini, 2009). Além de a ocorrência da depressão materna estar relacionada a efeitos adversos no desenvolvimento infantil, o caráter crônico ou recorrente da depressão e sua severidade são aspectos relacionados a maiores prejuízos. Crianças com mães deprimidas tendem a ter mais problemas de comportamento ou depressão quanto maior foi a severidade e a duração da depressão materna (Goodman, 2007; Hammen \& Brennan, 2003).

Considerando o risco que a depressão materna constitui para o desenvolvimento infantil, a participação do pai nos cuidados com a criança tem sido considerada um possível fator de proteção contra problemas emocionais e comportamentais na infância. Alguns estudos têm indicado que quando os pais de crianças de mães deprimidas se envolvem no cuidado ou nas atividades cotidianas dos filhos, os efeitos adversos da depressão materna sobre o desenvolvimento infantil tendem a ser reduzidos (Chang, Halpern, \& Kaufman, 2007; Letourneau, Duffett-Leger, \& Salmani, 2009; Mezulis, Hyde, \& Clark, 2004).

Embora não seja objetivo do presente estudo investigar a depressão paterna, alguns estudos indicam que a depressão materna e a paterna tendem a covariar e que a depressão paterna é mais comum entre o terceiro e o sexto mês de vida do bebê (Paulson \& Bazemore, 2010). É importan- te destacar ainda que a depressão paterna pode exacerbar os efeitos da depressão materna sobre o desenvolvimento infantil, assim como a saúde mental do pai, no contexto da depressão materna, tende a constituir um fator de proteção (Gere et al., 2013; Mezulis et al., 2004). Desse modo, a atuação do pai enquanto cuidador, especialmente quando ele não apresenta sintomas de depressão, parece reduzir os prejuízos causados pela interação da criança com uma mãe deprimida.

Quanto à investigação da participação do pai no cuidado dos filhos, este fenômeno tem sido pesquisado por meio de diferentes constructos, mas, particularmente, pelo uso do conceito de envolvimento paterno. Esse construto designa um fenômeno multidimensional, que considera tanto as práticas de socialização empregadas pelo pai na criação dos filhos, quanto seu estilo de participação nos cuidados diários e interação em atividades cotidianas com a criança (Marsiglio \& Pleck, 2005). Existem dois modelos teóricos acerca do envolvimento paterno. O primeiro, desenvolvido por Lamb, Pleck, Charnov e Levine (1985), compreende o envolvimento paterno a partir de três dimensões distintas: (a) interação, que designa o quanto o pai mantém contato direto e engajamento nas atividades de cuidado e lazer com seu filho; (b) disponibilidade, que se refere a quanto o pai está presente e disponível para a criança de forma física e psicológica e (c) responsabilidade, que contempla o fato de o pai garantir os recursos necessários para a manutenção da saúde, educação e bem estar geral da criança e participar ativamente de atividades ligadas a esses âmbitos (Lamb \& Tamis-Lemonda, 2010). O segundo modelo, desenvolvido por Pleck (2010) a partir da proposta anterior, envolve cinco dimensões com o objetivo de incluir efetivamente a análise das práticas de socialização paternas e da dimensão afetiva ou emocional da interação pai-criança, que não apareciam de modo tão claro no modelo original. No presente estudo, foi utilizado o modelo de Lamb et al. (1985), por tratar-se do modelo mais frequente na literatura, facilitando, portanto, a comparação com outros estudos da área.

Apesar de a relação entre depressão materna e o desenvolvimento infantil e do efeito modera- 
dor do envolvimento paterno estarem bem documentados na literatura (Allen \& Daly, 2007; Chang et al., 2007; Letourneau et al., 2009; Mezulis et al., 2004), a relação entre a depressão materna e o envolvimento paterno ainda não foi esclarecida. Considerando a função protetora que o envolvimento paterno pode ter sobre o desenvolvimento infantil, no contexto da depressão materna, é essencial que se investigue se a participação do pai nos cuidados com a criança aumenta ou pode ser reduzida, de acordo com os indicadores de depressão da mãe. O estudo de Goodman (2008) encontrou uma correlação negativa entre a depressão pós-parto e as habilidades do pai de responder sensivelmente à criança durante a interação entre o segundo e o terceiro mês de vida. Além disso, a depressão pós-parto esteve positivamente associada aos níveis de interação disfuncional entre pai e criança e ao estresse paterno. Nessa mesma perspectiva, o estudo de Séjourné, Vaslot, Beaumé, Goutaudier e Chabrol (2012) mostrou que quanto maior a intensidade da depressão materna, avaliada entre o segundo e o quinto dia após o nascimento, maior a falta de envolvimento paterno no segundo mês de vida do bebê. Smith e Howard (2008), visando a compreender possíveis mudanças nas relações entre essas duas variáveis, ao longo dos dois primeiros anos de vida da criança, realizaram um estudo longitudinal, envolvendo diferentes medidas, tanto da depressão materna, como da participação do pai, avaliada pelo construto de "suporte paterno". Os resultados confirmaram a relação entre maior suporte paterno e menor sintomatologia depressiva. Além disso, o estudo mostrou que tanto os sintomas de depressão quanto o suporte paterno diminuíram ao longo dos dois anos. O estudo realizado no Brasil por Mendonça, Bussab, Rodrigues, Siqueira e Cossette (2012), também aponta para a mesma direção. Foram entrevistados mães e pais aos três meses e aos três anos de idade dos filhos. A partir da percepção materna sobre o envolvimento paterno, o envolvimento considerado como abaixo do ideal pelas mães esteve associado à presença de depressão materna. No entanto, os pais relataram mais envolvimento aos três anos da criança quando a mãe teve depressão pós-par- to (no terceiro mês). Por outro lado, o estudo de Mezulis et al. (2004) não verificou qualquer tipo de relação entre a depressão materna, investigada em dois períodos da vida da criança (durante o primeiro ano de vida e no jardim de infância) e o afeto e o controle paternos.

Os estudos que avaliaram as relações entre a depressão materna e o envolvimento paterno indicaram, predominantemente, relações inversas entre as duas variáveis, como os estudos de Goodman (2008), Séjourné et al. (2012) e Smith e Howard (2008), que investigaram, a partir da percepção das mães, o envolvimento paterno no contexto da depressão materna. Tais achados sugerem um maior risco de que filhos de mães deprimidas experimentem menor contato com o pai, ou que essas interações tenham menor qualidade. Contudo, um dos estudos revisados não verificou relações entre as duas variáveis (Mezulis et al., 2004) e um estudo brasileiro detectou maior participação do pai aos três anos de vida, quando a mãe apresentou depressão no terceiro mês da criança (Mendonça et al., 2012). A inconsistência dos achados pode estar relacionada a aspectos teórico-metodológicos, como a diversidade de constructos e medidas utilizadas na avaliação de ambas as variáveis, ou ao tipo de informante, isto é, se o envolvimento paterno foi avaliado pelo relato da mãe ou do próprio pai. Da mesma forma, esses achados apontam ainda para possíveis diferenças culturais ou mudanças nas relações em questão, ao longo do tempo ou do desenvolvimento da criança. Por exemplo, o estudo de Goodman, Lusby, Thompson, Newport e Stowe (2014), que teve o objetivo de examinar associações entre depressão materna e envolvimento paterno, ilustra as modificações das associações ao longo do tempo. Os autores verificaram, nos primeiros seis meses de vida da criança, uma associação positiva entre depressão materna e envolvimento paterno, com posterior mudança para uma associação negativa que persistiu entre os sete e os 12 meses de vida da criança.

A literatura indica os efeitos negativos da depressão materna sobre $\mathrm{o}$ desenvolvimento infantil e, ao mesmo tempo, o possível caráter protetivo do envolvimento paterno nesse contexto (Chang et al., 2007; Letourneau et al., 2009; 
Mezulis et al., 2004). Por essas razões, é necessário investigar essa problemática, especialmente através de estudos que busquem descrever a associação entre a depressão materna e o envolvimento paterno ao longo do tempo.

Assim, o presente estudo teve como objetivo avaliar as relações entre indicadores de depressão da mãe e sua percepção acerca do envolvimento paterno. As hipóteses testadas foram as seguintes: (a) quanto maiores os escores de depressão materna no primeiro e no $18^{\circ}$ mês da criança, menor a frequência de relatos da mãe acerca do envolvimento paterno aos 30 meses da criança, nas três dimensões avaliadas; (b) mães com nível de depressão clínico no primeiro e no $18^{\circ}$ mês da criança tenderiam a relatar menos envolvimento paterno aos 30 meses da criança quando comparadas às mães sem depressão.

\section{Participantes}

\section{Método}

Participaram deste estudo 19 mães de crianças do sexo masculino. A seleção da amostra ocorreu em quatro maternidades públicas da cidade de Salvador quando as participantes esta- vam no terceiro trimestre da gestação. As mães deste estudo foram selecionadas dentre as participantes de um estudo longitudinal sobre determinantes do comportamento antissocial na infância (Alvarenga, Dazzani, Lordelo, Alfaya, \& Piccinini, 2007), motivo pelo qual todas as crianças eram do sexo masculino, visto que os meninos constituem grupo de risco para esse tipo de comportamento.

Os dados sociodemográficos das participantes estão descritos na Tabela 1. A idade das mães variou entre 26 e 42 anos, sendo a média de 32,05 $(D P=5,05)$. A variação de idade dos pais esteve entre 23 e 48 anos, com média de $33,84(D P=6,39)$. Quanto ao nível de escolaridade, as mães $(M=11,56, D P=2,38)$ e os pais $(M=11,94, D P=1,92)$ tinham a média de anos de estudo equivalente ao ensino médio completo ou incompleto. A renda familiar média foi de $\mathrm{R} \$ 1.442,82(D P=1.158,23)$, variando de $\mathrm{R} \$ 450,00$ a $\mathrm{R} \$ 5.450,00$. Do total das 19 mães, dez não possuíam atividade remunerada, enquanto somente um dos pais não exercia atividade remunerada. De todos os pais, somente dois não coabitavam com a criança.

Tabela 1

Dados Sociodemográficos dos Participantes aos 30 Meses das Crianças

\begin{tabular}{llcccc}
\hline \multicolumn{1}{c}{ Variáveis } & \multicolumn{1}{c}{ Níveis } & \multicolumn{3}{c}{ Valores } \\
\cline { 3 - 5 } & & $n$ & $\%$ & $M$ & $D P$ \\
\hline Idade da mãe (anos) & & & 32,05 & 5,05 \\
Idade do pai (anos) & & & 33,84 & 6,39 \\
Escolaridade da mãe (anos) & & & 11,56 & 2,38 \\
Escolaridade do pai (anos) & & & 11,94 & 1,92 \\
Renda familiar (Reais) & & & & $1.442,82$ & $1.158,23$ \\
Ocupação da mãe & Trabalha fora de casa & 9 & 47,4 & & \\
& Não trabalha fora de casa & 10 & 52,6 & & \\
Ocupação do pai & Trabalha fora de casa & 18 & 94,7 & & \\
Coabitação parental & Não trabalha fora de casa & 1 & 5,3 & \\
& Sim & 17 & 89,5 & & \\
& Não & 2 & 10,5 & \\
\hline
\end{tabular}




\section{Delineamento e Procedimentos}

Utilizou-se um delineamento correlacional de caráter longitudinal. As mães foram contatadas e convidadas a participar do estudo enquanto realizavam acompanhamento pré-natal em quatro maternidades públicas de Salvador. As gestantes foram informadas sobre os objetivos e os procedimentos do estudo e aquelas que concordaram em participar assinaram o Termo de Consentimento Livre e Esclarecido. Posteriormente, foram realizadas três visitas domiciliares, quando os bebês estavam no primeiro, $18^{\circ}$ e $30^{\circ}$ mês de vida, para aplicação de questionários e entrevistas com as mães. No primeiro mês de vida do bebê e quando a criança estava com um ano e meio de idade, a mãe respondeu ao Inventário de Depressão de Beck (BDI; Beck \& Steer, 1993). Destaca-se que três participantes não responderam ao BDI no $18^{\circ}$ mês, mas que responderam às entrevistas do $30^{\circ}$ mês. Quando as crianças estavam com dois anos e meio, todas as mães foram entrevistadas sobre sua percepção acerca do envolvimento paterno e sobre os dados sociodemográficos da família. O estudo foi submetido e aprovado pelo Comitê de Ética em Pesquisa da Faculdade de Filosofia e Ciências Humanas da Universidade Federal da Bahia (UFBA), em 27 de setembro de 2007.

\section{Instrumentos}

Ficha de Dados Sociodemográficos. Elaborada para investigar aspectos sociodemográficos como idade, escolaridade e realização de atividade remunerada de ambos os pais, assim como a renda familiar. Esse instrumento foi respondido pelas mães quando as crianças estavam no $30^{\circ}$ mês de vida.

Inventário Beck de Depressão (BDI; Beck $\&$ Steer, 1993). Trata-se de uma escala sintomática de autorrelato, composta por 21 itens com diferentes alternativas de resposta a respeito de como o participante tem se sentido na última semana e no dia em que responde ao inventário. Em cada item, o participante deve concordar com uma afirmativa em um conjunto com quatro sentenças associadas com números de zero a três. São exemplos de sentenças: (0) Não me sinto triste; (1) Eu me sinto triste; (2) Estou sempre triste e não consigo sair disto; (3) Estou tão triste ou infeliz que não consigo suportar. Através da soma dos escores dos itens, obtém-se um escore total, que estabelece a intensidade da depressão, classificada nos seguintes níveis: mínimo (até 11 pontos), leve (de 12 a 19 pontos), moderado (de 20 a 35 pontos) ou grave (acima de 36 pontos). Para o presente estudo, foi utilizada a versão em português do inventário cuja consistência interna é de 0,84 e a correlação entre teste e reteste de 0,40 ( $p<0,001$; Cunha, 2001). Destaca-se que o BDI é uma medida dos indicadores de depressão, não oferecendo um diagnóstico clínico de depressão.

Entrevista sobre a Percepção da Mãe acerca do Envolvimento Paterno (Alvarenga, 2010). Refere-se a uma entrevista semiestruturada que investigou a participação do pai nos cuidados e atividades com a criança durante a semana e nos fins de semana, a partir do relato materno. Dentre os aspectos abordados na entrevista, destacam-se: a participação do pai nos cuidados com a saúde do filho, bem como seu envolvimento na educação formal e informal, e na socialização dos filhos em episódios disciplinares e situações de conflito. São exemplos de perguntas da entrevista: "O pai acompanha a criança nas consultas ao médico ou vacinação?”, "O pai ensina coisas a criança?", "Quando o (nome da criança) fica teimoso ou faz birra porque quer alguma coisa, o pai faz alguma coisa? O que ele faz nessas situações?".

\section{Procedimento de Análise dos Dados}

As entrevistas sobre a Percepção da Mãe acerca do Envolvimento Paterno foram submetidas à análise de conteúdo (Bardin, 1977) realizada por dois codificadores independentes que receberam treinamento de 20 horas. O coeficiente Kappa obtido foi de 0,92. Após a classificação das unidades de análise nas categorias identificadas, a frequência de cada uma das categorias e subcategorias foi calculada e esses valores foram utilizados nas análises estatísticas descritas a seguir.

Para as análises estatísticas, foi utilizado o software Statistical Package for Social Science (SPSS 20.0). As frequências e os níveis dos escores de depressão da mãe, baseados nos indicadores do BDI foram obtidos por meio de 
estatísticas descritivas. O teste de Correlação de Spearman foi utilizado para verificar a relação entre as variáveis sociodemográficas, os indicadores de depressão materna e as categorias de envolvimento paterno. O teste Mann-Whitney foi realizado para verificar se existiam diferenças quanto à percepção sobre o envolvimento paterno, entre as mães do grupo clínico e não-clínico, classificadas a partir dos escores indicadores de depressão do BDI.

\section{Resultados}

As frequências dos níveis de depressão baseados nos indicadores do BDI são apresentados na Tabela 2. O nível mínimo equivale à ausência de depressão. No primeiro mês de vida da criança, dez mães $(52,5 \%)$ apresentaram intensidade mínima de depressão, quatro $(21,1 \%)$ tiveram intensidade leve e cinco (26,3\%) tiveram intensidade moderada. Enquanto no $18^{\circ}$ mês, sete mães $(36,8 \%)$ apresentaram escores no nível mínimo, seis $(31,6 \%)$ no nível leve e três $(15,8 \%)$ no nível moderado. Verifica-se que houve uma redução dos casos mínimos e moderados entre o primeiro e o $18^{\circ}$ mês, com o aumento de casos com intensidade leve de depressão no segundo momento. Verificou-se também que nenhum dos casos foi classificado no nível grave.

Tabela 2

Frequências dos Níveis dos Escores de Depressão Baseados nos Indicadores do BDI

\begin{tabular}{|c|c|c|c|c|}
\hline Período & Mínimo & Leve & Moderado & Grave \\
\hline $1^{\circ}$ mês & $10(52,6 \%)$ & $4(21,1 \%)$ & $5(26,3 \%)$ & $0(0 \%)$ \\
\hline $18^{\circ}$ mês & $7(36,8 \%)$ & $6(31,6 \%)$ & $3(15,8 \%)$ & $0(0 \%)$ \\
\hline
\end{tabular}

Os relatos das mães investigados através da Entrevista sobre a Percepção da Mãe acerca do Envolvimento Paterno foram agrupados em três grandes categorias, baseadas na proposta de Lima (2008) e no modelo de envolvimento paterno de Lamb et al. (1985). As categorias foram denominadas interação pai-criança, disponibilidade paterna e responsabilidade paterna. A primeira categoria, interação pai-criança, compreende duas subcategorias que abordam: (a) lazer e (b) cuidado. A primeira subcategoria inclui relatos acerca da interação pai-criança em situações de lazer como passear de bicicleta, desenhar ou assistir televisão. São exemplos de relatos classificados nesta subcategoria: “. . . o tempo todo jogando videogame com o pai" e “. . bota DVD, assistem juntos". A segunda subcategoria engloba atividades de cuidados, como higiene ou alimentação, realizadas de maneira direta ou indireta com a criança, por exemplo, dar banho ou cozinhar para o filho. Como exemplo de relato classificado nessa subcategoria, tem-se "[ele] dá banho, dá comida e faz comida".
A segunda categoria, disponibilidade paterna, refere-se a relatos sobre a disponibilidade do pai (física ou psicológica), quando solicitado, para realizar todos os tipos de tarefas com a criança, bem como a disposição para lidar ou ajudar a mãe a gerenciar as situações difíceis e é constituída por duas subcategorias: (a) cuidado, lazer e saúde e (b) situações difíceis. Nesta categoria, foram incluídos os relatos sobre o pai parar de realizar uma atividade para ajudar a mãe nos cuidados ou na interação com a criança. São exemplos de situações inclusas nesta categoria: o pai ficar sozinho com a criança ou fazer comida quando necessário ou manejar situações em que a criança não queira comer ou a faça birra. A subcategoria cuidado, lazer e saúde envolve situações nas quais os pais não costumam fazer determinadas tarefas, mas se dispõem a realizá-las quando solicitados. Como exemplo de relatos desta subcategoria, cita-se: ". . . sábado ele fica com ele de manhã, porque eu tenho curso". Já na subcategoria situações difíceis, incluem-se ações que os pais não costumam realizar, mas 
se mantêm dispostos a realizá-las, demonstrando disposição para resolver ou ajudar a resolver as situações que a mãe não consegue resolver sozinha. São exemplos desta subcategoria: “. . . Ele faz aviãozinho, faz... ele ajuda nesse sentido. . . . Quando ele está em casa ele ajuda, ele fala: 'Ah! Essa comida gostosa"”.

Por fim, a terceira categoria, responsabilidade paterna, corresponde aos relatos acerca da responsabilidade do pai em atividades que envolvem participar diretamente dos cuidados à saúde e à educação da criança. São exemplos de relatos desta categoria: o pai levar a criança ao pediatra ou dar remédios a ela, bem como levar ou buscar a criança na escola, ou ainda, ensinar algo a ela. Esta categoria possui duas subcategorias: (a) saúde e (b) educação. Na subcategoria saúde, foram incluídos relatos como: ". . . se for uma campanha de vacina . . quem leva é ele". Por último, a subcategoria educação, tem como

Tabela 3

Frequências Médias e Desvios-Padrão das Categorias de Envolvimento Paterno

\begin{tabular}{|c|c|}
\hline Categorias & $M(D P)$ \\
\hline Interação pai-criança & $14,89(6,72)$ \\
\hline Lazer & $4,84(3,18)$ \\
\hline Cuidado & $10,05(4,77)$ \\
\hline Disponibilidade paterna & $0,84(1,17)$ \\
\hline Cuidado, lazer e saúde & $0,68(1,00)$ \\
\hline Situações difíceis & $0,16(0,38)$ \\
\hline Responsabilidade paterna & $3,11(2,26)$ \\
\hline Saúde & $1,42(1,12)$ \\
\hline Educação & $1,68(1,83)$ \\
\hline
\end{tabular}

Analisou-se, através de uma correlação bivariada não-paramétrica (Spearman), a relação entre as variáveis sociodemográficas, os indicadores de depressão materna no primeiro e no $18^{\circ}$ mês de vida da criança e as categorias de envolvimento paterno do $30^{\circ}$ mês da criança. A Tabela 4 apresenta os valores das correlações entre as variáveis mencionadas. Os indicadores de depressão não estiveram correlacionados com os dados sociodemográficos investigados. Entre os exemplo dos relatos: “. . . ele pega A. na escola. .. , pega revista, fica falando as figuras".

As frequências médias e os desvios-padrão das categorias de envolvimento paterno relatadas pelas mães são apresentados na Tabela 3. A categoria que teve maior frequência média nos relatos maternos foi a de interação pai-criança $(M=14,89, D P=6,72)$, com a subcategoria interação em atividades de cuidado possuindo maior frequência nos relatos maternos $(M=10,05$, $D P=4,77)$. A categoria disponibilidade paterna apresentou uma frequência média inferior a um relato por participante $(M=0,84, D P=1,17)$, de modo que a disponibilidade em cuidado, lazer e saúde é mais frequente $(M=0,68, D P=1,00)$. A responsabilidade paterna, por sua vez, foi a segunda categoria mais frequente $(M=3,11$, $D P=2,26$ ), na qual os relatos de referentes à saúde $(M=1,42, D P=1,12)$ e à educação da criança $(M=1,68, D P=1,83)$ obtiveram frequências médias semelhantes. 
sentido, quanto maior a renda familiar, mais frequentes os relatos maternos sobre a responsabilidade do pai no que tange à educação da criança.

No que se refere às relações entre os indicadores de depressão materna e as categorias de envolvimento paterno, identificou-se uma correlação positiva moderada significativa entre os in- dicadores de depressão ao primeiro mês de vida do bebê e a categoria de disponibilidade paterna no trigésimo mês de vida da criança $(r=0,44, p$ $=0,03)$. Os indicadores de depressão ao primeiro mês também apresentaram uma correlação positiva moderada significativa com a subcategoria disponibilidade em cuidado, lazer e saúde ( $r=$ $0,55, p=0,01)$.

\section{Tabela 4}

Correlação entre Dados Sociodemográficos, Indicadores de Depressão Materna e Envolvimento Paterno

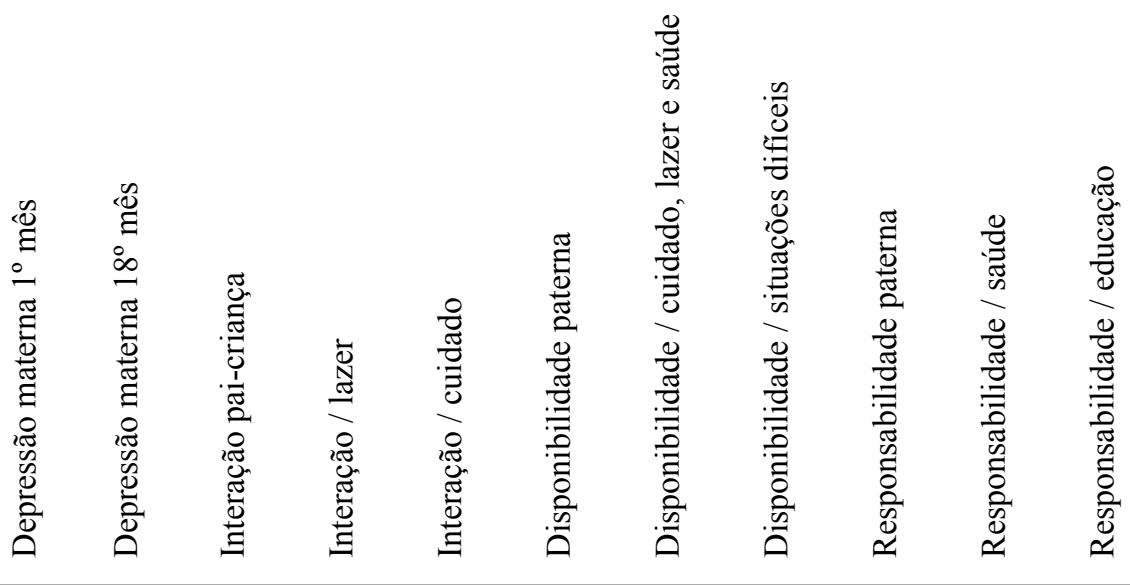

Dados sociodemográficos

Idade da mãe

$-0,04-0,32$

$-0,35$

$-0,15$

$-0,38 \quad-0,18$

$-0,13$

$-0,30$

$-0,27$

$-0,13$

$-0,27$

Escolaridade da mãe

$\begin{array}{lll}-0,06 & -0,10 & -0,28\end{array}$

$-0,16$

$-0,14$

$-0,35$

$-0,27 \quad-0,14$

$-0,17 \quad-0,30 \quad-0,11$

Idade do pai

$\begin{array}{lll}-0,07 & -0,27 & -0,36\end{array}$

$\begin{array}{llll}-0,29 & -0,27 & 0,25 & 0,56^{* *}\end{array}$

$-0,33$

$-0,23$

$-0,22-0,15$

$-0,16 \quad-0,14 \quad-0,08$

Escolaridade do pai

$0,34 \quad-0,42$

$0,28 \quad 0,11$

0,32

$-0,13$

$-0,19 \quad 0,04$

0,35

$-0,12 \quad 0,39$

Renda familiar

$$
0,52^{*}
$$

$$
0,08 \quad-0,20
$$$$
0,12
$$$$
0,44^{*}
$$$$
0,55^{*}
$$

0,07

$-0,09$

$-0,01 \quad-0,07$

$18^{\circ}$ mês

$$
0,14 \quad 0,16
$$

0,11

0,19

0,36

$-0,16$

$-0,18 \quad-0,13 \quad-0,22$

Envolvimento paterno

Interação pai-criança

$$
\begin{array}{cccccccc}
0,69^{* *} & 0,90^{* *} & 0,67^{* *} & 0,56^{* *} & 0,29 & 0,41^{*} & 0,18 & 0,36 \\
0,36 & 0,38 & 0,29 & 0,21 & 0,16 & -0,08 & 0,19 \\
& 0,59^{* *} & 0,48^{*} & 0,25 & 0,42^{*} & 0,32 & 0,32 \\
& & 0,93^{* *} & 0,53^{* *} & 0,25 & 0,27 & 0,20 \\
& & & 0,25 & 0,08 & 0,14 & 0,06 \\
& & & & 0,53^{* *} & 0,37 & 0,52^{*} \\
& & & & & & 0,54^{* *} & 0,89^{* *} \\
& & & & & & 0,13
\end{array}
$$

Disponibilidade / cuidado, lazer e saúde

Disponibilidade / situações difíceis

Responsabilidade / saúde

Responsabilidade / educação

$* p \leq 0,05 ; * * p \leq 0,01$. 
Os dados da Tabela 5 complementam as análises de correlação apresentadas anteriormente. Na Tabela 5, comparou-se, quanto às categorias de envolvimento paterno, o grupo não-clínico (BDI $\leq 11)$ e o clínico (BDI $\geq 12)$, com dados referentes aos indicadores de depressão no primeiro e no $18^{\circ}$ mês através do teste Mann-Whitney. A categoria disponibilidade paterna e a subcategoria disponibilidade em cuidado, lazer e saúde apresentaram diferenças significativas entre os grupos. $\mathrm{O}$ grupo clínico teve maiores valores em comparação ao não-clínico $(p=0,04)$ na categoria disponibilidade paterna e também obteve valores maiores, quando comparado ao não-clínico $(p=0,02)$, para a subcategoria disponibilidade em cuidado, lazer e saúde.

\section{Tabela 5}

Comparação entre os Grupos Clínicos e Não-Clínicos de Depressão Materna quanto às Categorias de Envolvimento Paterno com o Teste Mann-Whitney

\begin{tabular}{|c|c|c|c|c|c|c|c|c|}
\hline \multirow{2}{*}{$\begin{array}{c}\text { Categorias } \\
\text { de Envolvimento } \\
\text { Paterno }\end{array}$} & \multicolumn{2}{|c|}{ Depressão materna $1^{\circ}$ mês } & \multirow[b]{2}{*}{$U$} & \multirow[b]{2}{*}{$p$} & \multicolumn{2}{|c|}{ Depressão materna $18^{\circ}$ mês } & \multirow[b]{2}{*}{$U$} & \multirow[b]{2}{*}{$p$} \\
\hline & $\begin{array}{l}\text { Não-Clínico } \\
\qquad \begin{array}{c}(n=9) \\
M(D P)\end{array}\end{array}$ & $\begin{array}{l}\text { Clínico } \\
(n=10) \\
M(D P)\end{array}$ & & & $\begin{array}{c}\text { Não-Clínico } \\
\qquad(n=6) \\
M(D P)\end{array}$ & $\begin{array}{l}\text { Clínico } \\
(n=10) \\
M(D P)\end{array}$ & & \\
\hline Interação pai-criança & $14,11(6,92)$ & $15,60(6,82)$ & 35,00 & 0,45 & $13,67(8,45)$ & $15,10(6,54)$ & 22,00 & 0,35 \\
\hline Lazer & $4,56(3,00)$ & $5,10(3,48)$ & 43,50 & 0,90 & $4,00(2,68)$ & $5,00(3,80)$ & 20,50 & 0,25 \\
\hline Cuidado & $9,56(5,10)$ & $10,50(4,67)$ & 38,00 & 0,60 & $9,67(6,50)$ & $10,10(3,54)$ & 25,00 & 0,54 \\
\hline Disponibilidade paterna & $0,22(0,44)$ & $1,40(1,35)$ & 19,50 & $0,04 *$ & $0,50(0,55)$ & $0,90(1,45)$ & 28,00 & 0,76 \\
\hline $\begin{array}{l}\text { Cuidado, lazer } \\
\text { e saúde }\end{array}$ & $0,11(0,33)$ & $1,20(1,14)$ & 17,00 & $0,02 *$ & $0,33(0,52)$ & $0,80(1,23)$ & 24,50 & 0,47 \\
\hline Situações difíceis & $0,11(0,33)$ & $0,20(0,42)$ & 41,00 & 0,78 & $0,17(0,41)$ & $0,10(0,32)$ & 30,50 & 0,92 \\
\hline $\begin{array}{l}\text { Responsabilidade } \\
\text { paterna }\end{array}$ & $3,67(2,78)$ & $2,60(1,65)$ & 37,00 & 0,55 & $3,33(2,66)$ & $3,00(2,36)$ & 28,00 & 0,76 \\
\hline Saúde & $1,78(1,39)$ & $1,10(0,74)$ & 34,50 & 0,40 & $2,00(1,79)$ & $1,20(0,63)$ & 26,50 & 0,61 \\
\hline Educação & $1,89(2,26)$ & $1,50(1,43)$ & 44,00 & 0,97 & $1,33(1,21)$ & $1,80(2,25)$ & 25,50 & 0,54 \\
\hline
\end{tabular}

$* p \leq 0,05$.

\section{Discussão}

A hipótese deste estudo era de que quanto maiores os indicadores de depressão materna no primeiro e no $18^{\circ}$ mês da criança, menor seria a frequência de relatos da mãe de envolvimento paterno aos 30 meses nas três dimensões avaliadas. Outra hipótese era de que mães com nível clínico de depressão nesses dois períodos tenderiam a relatar menos envolvimento paterno aos 30 meses da criança, quando comparadas com mães sem depressão. Ambas as hipóteses foram refutadas. A única correlação constatada entre as duas variáveis investigadas foi direta, indicando que quanto maiores os escores de depressão pós-parto (primeiro mês), mais frequentes os relatos sobre a disponibilidade do pai aos trinta meses, particularmente, para atender a criança em cuidados de rotina, com a saúde, e em atividades de lazer. As análises que compararam mulheres com níveis clínicos de depressão e mulheres sem depressão, confirmaram esses achados. Não foi verificada nenhuma correlação entre as dimensões do envolvimento paterno e a depressão materna no $18^{\circ}$ mês de vida da criança.

As relações encontradas entre a depressão pós-parto e a maior disponibilidade do pai para interagir e cuidar da criança, embora não fossem 
previstas de acordo com a literatura revisada, merecem ser exploradas. A dimensão disponibilidade refere-se à presença do pai e à sua possibilidade de auxiliar a mãe e participar dos cuidados com a criança sempre que necessário (Lamb et al., 1985). No presente estudo, essa dimensão foi investigada concretamente a partir dos relatos maternos que indicavam que o pai só realizava ou participava de determinada atividade de cuidado ou lazer, quando solicitado pela mãe a assim fazê-lo. Portanto, as correlações positivas que foram encontradas indicam que os sintomas de depressão pós-parto, embora não tenham aumentado ou diminuído efetivamente a participação e o envolvimento do pai nos cuidados com a criança, podem tê-lo tornado mais sensível à necessidade de apoio da mãe, e consequentemente, mais propenso a auxiliá-la participando dos cuidados com a criança, desde que fosse solicitado a fazê-lo. Esse achado pode estar indicando o impacto da depressão pós-parto sobre o envolvimento paterno, embora essa relação pareça depender da solicitação de auxílio por parte da mãe.

Outro aspecto que chama atenção é o fato de que apenas a depressão pós-parto esteve positivamente correlacionada à disponibilidade, enquanto a depressão no $18^{\circ}$ mês não esteve correlacionada nem a essa, nem a qualquer outra dimensão avaliada. A lacuna ocasionada pela ausência de respostas de três mães sobre a depressão no $18^{\circ}$ mês pode ter colaborado para a ausência de correlação com as dimensões de envolvimento paterno. No entanto, o estudo de Mendonça et al. (2012), encontrou resultado semelhante, mostrando que o envolvimento paterno aos três anos da criança foi maior quando a mãe teve depressão no terceiro mês. É possível que sintomas depressivos maternos presentes logo após o nascimento da criança, ou nos primeiros meses, tornem o pai mais disponível, por este se tratar de um período extremamente sensível e de transição (Menezes \& Lopes, 2007). Neste período, a relação do casal passa por profundas mudanças, e a própria satisfação e alegria com a chegada do bebê, poderiam sensibilizar o pai e aumentar seu envolvimento com o filho quando a mãe está deprimida. É interessante perceber que tanto no presente estudo, como no estudo de
Mendonça et al. (2012) os efeitos da depressão pós-parto sobre o envolvimento paterno foram constatados em períodos posteriores, ou seja, em torno do terceiro e quarto ano de vida. Assim, é possível ainda que, em decorrência dos cuidados que um recém-nascido demanda e dos sintomas depressivos, as mulheres com mais indicadores de depressão tenham solicitado com mais frequência o auxílio dos pais no início da vida criança, estabelecendo um padrão de cuidado na família que se manteve ao longo do tempo.

De qualquer modo, é preciso considerar o fato de que diferentes estudos internacionais revisados respaldavam a previsão de correlações negativas entre a depressão materna e o envolvimento paterno (Goodman, 2008; Séjourné et al., 2012; Smith \& Howard, 2008). Nesse sentido, é importante que estudos brasileiros continuem investigando essa problemática, para que seja possível explorar melhor a hipótese acerca do impacto positivo da depressão materna sobre o envolvimento paterno, e se essa relação pode estar permeada por fenômenos típicos da nossa cultura, já que os estudos internacionais não confirmam essa tendência.

Houve também correlações entre variáveis sociodemográficas e o envolvimento paterno. A escolaridade do pai e a renda familiar estiveram positivamente correlacionadas, respectivamente, com as categorias interação em lazer e responsabilidade com a educação. No que tange à relação entre escolaridade e interação em lazer, o estudo de Amato e Rivera (1999), que visou a investigar as relações entre envolvimento paterno e problemas de comportamento em crianças e adolescentes, constatou que o nível de escolaridade de ambos os pais esteve positivamente correlacionado ao envolvimento paterno. O estudo de Yoshida (2012) também obteve resultados semelhantes, que revelaram que quanto maior o nível de instrução dos pais, mais frequentes foram os seus relatos sobre brincadeiras e cuidados físicos com os filhos. Neste estudo, essa relação esteve associada à presença de pelo menos um filho do sexo masculino, o que chama atenção também para possíveis relações entre o envolvimento paterno e o sexo da criança. Nesse sentido, é importante destacar que o presente estudo investigou uma 
amostra composta exclusivamente por mães de meninos e que essa característica pode também estar relacionada aos achados obtidos.

De qualquer modo, supõe-se que pais com maior escolaridade têm maiores possibilidades de acesso a informações relevantes quanto às condutas apropriadas para favorecer o desenvolvimento socioemocional infantil. Ainda assim, é interessante notar que o impacto da escolaridade paterna apareceu apenas na categoria interação em lazer, porém, não se manifestou em outras dimensões importantes, como a interação em cuidados (ex. dar banho ou alimentar a criança). É possível que os valores e condutas associadas à representação do papel do pai em nossa cultura, ainda estejam muito vinculadas a atividades lúdicas e de lazer com a criança e não aos cuidados básicos, que são frequentemente atribuídos à mãe. Esse padrão cultural também foi constatado por Monteiro, Veríssimo, Santos e Vaughn (2008), em uma pesquisa sobre envolvimento paterno e apego em famílias portuguesas, que encontrou uma associação positiva entre escolaridade paterna e interação em atividades de lazer.

Quanto à correlação positiva entre renda familiar e responsabilidade com a educação, a literatura é controversa. Por um lado, sugere-se que famílias com maior renda familiar têm pais mais envolvidos no cuidado dos seus filhos. Por exemplo, o estudo de Souza e Benetti (2008), com pais desempregados, verificou que os pais que possuíam maior renda, em decorrência de contribuição financeira fornecida pela família ou por outras fontes, eram mais envolvidos com os seus filhos quando comparados com os pais desempregados que não contavam com suporte financeiro. Por outro lado, encontra-se também na literatura uma correlação negativa entre envolvimento paterno e renda familiar. Por exemplo, Rienks, Wadsworth, Markman, Einhorn e Etter (2011) verificaram relação contrária em famílias populares urbanas dos Estados Unidos, revelando que quanto menor a renda familiar, mais envolvidos eram os pais com seus filhos. No que se refere ao presente estudo, dadas as características e a definição da dimensão responsabilidade, é possível que pais de famílias com maior renda, possuam uma rotina menos extenuante e, assim, possam contribuir de forma mais ativa, se preocupando com a saúde e a educação da criança e participando nesses domínios da vida dos filhos. No entanto, pesquisas futuras poderão aprofundar a discussão das relações entre renda familiar e envolvimento paterno.

Outra contribuição deste estudo é o compartilhamento de procedimentos e resultados de uma pesquisa em Psicologia do Desenvolvimento no Brasil com caráter longitudinal. Em uma revisão das produções de Psicologia do Desenvolvimento Infantil, nos principais periódicos nacionais, entre os anos de 2001 e 2010, Becker, Bandeira, Ghilardi, Hutz e Piccinini (2013) destacaram que a maioria dos estudos utilizou delineamento transversal apesar da relevância e adequação dos delineamentos longitudinais observados na literatura internacional em Psicologia do Desenvolvimento Infantil. Dadas as dificuldades operacionais encontradas no Brasil para se realizar pesquisas com esse tipo de delineamento, como dificuldades para o recrutamento da amostra e para a permanência dos participantes, descrever os procedimentos e os resultados como os deste estudo podem oferecer auxílio para os pesquisadores da área.

Por outro lado, o presente estudo também tem limitações. Com relação à avaliação do envolvimento paterno, os resultados levaram em conta a percepção da mãe como única informante. Nas pesquisas que compararam os relatos de pais e mães sobre envolvimento paterno, nota-se que os relatos maternos tendem a atribuir menor envolvimento ao pai, quando comparados aos relatos dos próprios pais (Mikelson, 2008; Prado, Piovanotti, \& Vieira, 2007). As diferenças entre os informantes, podem constituir um obstáculo para a interpretação dos estudos na área, pois oferecem maiores desafios aos pesquisadores na identificação da acurácia dos relatos. Soma-se a isso, o fato de que a depressão materna pode causar distorções na percepção da mulher acerca da participação do pai, tornando-a mais negativa. Há estudos que mostram que pessoas deprimidas tendem a perceber eventos de vida como mais negativos do que pessoas sem depressão (APA, 2013; Mendonça et al., 
2012), assim como há estudos que mostram que a depressão materna pode interferir no relato das mães acerca dos problemas de comportamento das crianças, elevando os escores (Boyle \& Pickles, 1997). Nesse sentido, é possível que os relatos sobre o envolvimento paterno também se mostrem enviesados, o que demandaria a participação de outros informantes sobre o envolvimento paterno. Nesse sentido, pesquisas futuras sobre o envolvimento paterno devem envolver diferentes informantes, tanto para que se possa examinar possíveis discrepâncias nos relatos materno e paterno, assim como para investigar o impacto da depressão materna sobre os relatos acerca do envolvimento paterno.

Por fim, a amostra foi composta por um número de casos bastante reduzido e foi comunitária e não clínica, envolvendo apenas mães com níveis mínimos, leves ou moderados de depressão. A ausência de participantes com depressão grave pode explicar, pelo menos em parte, a ausência de correlações negativas entre a depressão materna e o envolvimento paterno. Além disso, o envolvimento paterno foi avaliado somente aos trinta meses de vida da criança, impedindo que o impacto da depressão pós-parto e aos 18 meses fosse verificado em características do envolvimento paterno nesses mesmos períodos.

\section{Considerações Finais}

Em resumo, os achados do presente estudo, quanto à relação entre envolvimento paterno e depressão materna, indicam maior disponibilidade paterna no trigésimo mês de vida da criança, quando as mães apresentaram sintomas de depressão pós-parto, isto é, no primeiro mês de vida do bebê. Isso significa que os pais demonstraram maior envolvimento em cuidados e atividades com a criança em contextos em que as mães deprimidas solicitavam ou sinalizavam de alguma forma a necessidade de auxílio. A depressão materna aos 18 meses da criança não esteve relacionada a nenhuma das dimensões do envolvimento paterno.

Desse modo, ao contrário do que indicavam os achados da literatura revisada, o presente estudo não confirmou o valor preditivo da de- pressão materna sobre o nível de envolvimento dos pais com os filhos. De qualquer maneira, os achados do presente estudo, associados a resultados semelhantes em outra pesquisa realizada no Brasil, chamam a atenção para a necessidade de se investigar as relações entre a depressão pós-parto e o envolvimento paterno em nosso contexto cultural. Assim, sugere-se que novas pesquisas incluam a análise de amostras clínicas e investiguem o impacto do nível e da cronicidade dos sintomas depressivos sobre o envolvimento paterno, assim como do período em que a mãe apresenta esses sintomas, dando preferência a delineamentos longitudinais. Porém, é possível que a ausência de relações, no presente estudo, se deva ao reduzido número de casos analisados e, especialmente, à baixa frequência de casos de depressão moderada e à ausência de casos de depressão grave na amostra.

\section{Referências}

Allen, S., \& Daly, K. (2007). The effects of father involvement: An updated research summary of the evidence inventory. Guelph, Canada: Centre for Families, Work \& Well-Being, University of Guelph.

Alvarenga, P. (2010). Entrevista sobre a percepção da mãe acerca do envolvimento paterno. Projeto de pesquisa não publicado.

Alvarenga, P., Dazzani, M. V. M., Lordelo, E. R., Alfaya, C. A. S., \& Piccinini, C. A. (2007). Determinantes sociodemográficos, familiares e individuais do comportamento anti-social na infância. Manuscrito não publicado.

Alvarenga, P., Oliveira, J. M., \& Lins, T. (2012). O impacto da depressão materna em problemas internalizantes de pré-escolares. Aletheia, 38-39, 94-108.

Alvarenga, P., \& Palma, E. M. S. (2013). Indicadores de depressão materna e a interação mãe-criança aos 18 meses de vida. Psico, 44(3), 402-410.

Amato, P. R., \& Rivera, F. (1999). Paternal involvement and children's behavior problems. Journal of Marriage and the Family, 61(2), 375. doi:10.2307/353755

Associação Americana de Psiquiatria. (2013). Diagnostic and statistical manual of mental disorders ( $5^{\text {th }}$ ed.). Arlington, VA: Author. 
Bardin, L. (1977). Análise de conteúdo. Lisboa: Edições 70 .

Beck, A. T., \& Steer, R. A. (1993). Beck Depression Inventory: Manual. San Antonio, TX: Psychological Corporation.

Becker, S. M. S., Bandeira, C. de M., Ghilardi, R. B., Hutz, C. S., \& Piccinini, C. A. (2013). Psicologia do Desenvolvimento Infantil: Publicações nacionais na primeira década do Século XXI. Psico, 44(3), 372-381.

Boyle, M. H., \& Pickles, A. R. (1997). Influence of maternal depressive symptoms on ratings of childhood behavior. Journal of $A b$ normal Child Psychology, 25(5), 399-412. doi:10.1023/A:1025737124888

Chang, J. J., Halpern, C. T., \& Kaufman, J. S. (2007). Maternal depressive symptoms, father's involvement, and the trajectories of child problem behaviors in a US national sample. Archives of Pediatrics and Adolescent Medicine, 161(7), 697-703. doi:10.1001/archpedi.161.7.697

Cruz, E. B. D. S., Simões, G. L., \& Faisal-Cury, A. (2005). Rastreamento da depressão pós-parto em mulheres atendidas pelo Programa de Saúde da Família. Revista Brasileira de Ginecologia e Obstetrícia, 27(4), 181-188. doi:10.1590/ S0100-72032005000400004

Cunha, J. A. (2001). Manual da versão em português das Escalas Beck. São Paulo, SP: Casa do Psicólogo.

Field, T. (1995). Infants of depressed mothers. Infant Behavior and Development, 18, 1-13.

Field, T. (2010). Postpartum depression effects on early interaction, parenting, and safety practices: A review. Infant Behavior and Development, 33(1), 1-6. doi:10.1016/j.infbeh.2009.10.005

Frizzo, G. B., \& Piccinini, C. A. (2007). Depressão materna e a interação triádica pai-mãe-bebê. $P s i$ cologia: Reflexão e Crítica, 20(3), 351-360.

Gere, M. K., Hagen, K. A., Villabø, M. A., Arnberg, K., Neumer, S. P., \& Torgersen, S. (2013). Fathers' mental health as a protective factor in the relationship between maternal and child depressive symptoms. Depression and Anxiety, 30, 3138. doi:10.1002/da.22010

Goodman, J. H. (2008). Influences of maternal postpartum depression on fathers and on father-infant interaction. Infant Mental Health Journal, 29(6), 624-643. doi:10.1002/imhj.20199
Goodman, S. H. (2007). Depression in mothers. Annual Review of Clinical Psychology, 3, 107-135. doi:10.1146/annurev.clinpsy.3.022806.091401

Goodman, S. H., Lusby, C. M., Thompson, K., Newport, D. J., \& Stowe, Z. N. (2014). Maternal depression in association with fathers' involvement with theis infants: Spillover or compesation/buffering? Infant Mental Health Journal, 35(5), 495-508. doi:10.1002/imhj.21469

Gress-Smith, J. L., Luecken, L. J., Lemery-Chalfant, K., \& Howe, R. (2012). Postpartum depression prevalence and impact on infant health, weight, and sleep in low-income and ethnic minority women and infants. Maternal and Child Health Journal, 16(4), 887-893. doi:10.1007/s10995011-0812-y

Hammen, C., \& Brennan, P. (2003). Severity, chronicity, and timing of maternal depression and risk for adolescent offspring diagnoses in a community sample. Archives of General Psychiatry, 60, 253-260. doi:10.1001/archpsyc.60.3.253

Lamb, M. E., Pleck, J. H., Charnov, E. L., \& Levine, J. A. (1985). Paternal behavior in humans. American Zoologist, 25(3), 883-894.

Lamb, M. E., \& Tamis-Lemonda, C. S. (2010). The role of the father. In M. E. Lamb (Ed.), The role of the father in child development $\left(5^{\text {th }}\right.$ ed.). New York: Wiley.

Letourneau, N., Duffett-Leger, L., \& Salmani, M. (2009). The role of paternal support in the behavioural development of children exposed to postpartum depression. Canadian Journal of Nursing Research, 41(3), 86-106.

Lima, J. A. (2008). O tempo e as formas de envolvimento do pai em tarefas de socialização dos filhos em idade pré-escolar. Trabalho apresentado no $1^{\circ}$ Congresso Internacional em Estudos da Criança, Braga, Portugal. Recuperado em http:// sigarra.up.pt/fpceup/en/publs_pesquisa.show_ publ_file?pct_gdoc_id $=4843$

Loosli, L., \& Loureiro, S. R. (2010). Associação entre depressão materna e diferenças de gênero no comportamento de crianças: Uma revisão sistemática. Revista de Psiquiatria, 32(3), 94-101.

Lovejoy, M. C., Graczyk, P. A., O'Hare, E., \& Neuman, G. (2000). Maternal depression and parenting behavior: A meta-analytic review. Clinical Psychology Review, 20(5), 561-592. doi:10.1016/S0272-7358(98)00100-7 
Marsiglio, W., \& Pleck, J. H. (2005). Fatherhood and masculinities. In R. W. Connell, J. Hearn, \& M. Kimmel (Eds.), The handbook of studies on men and masculinities (pp. 249-269). Thousand Oaks, CA: Sage.

Mendonça, J. S., Bussab, V. S. R., Rodrigues, A., Siqueira, J., \& Cossette, L. (2012). Postpartum depression, father's involvement, and marital and co-parental relationships from mothers' and fathers' perspectives in a low-income Brazilian sample. Family Science, 3(3-4), 164-173. doi:10 $.1080 / 19424620.2012 .783423$

Menezes, C. C., \& Lopes, R. C. S. (2007). Relação conjugal na transição para a parental idade: Gestação até dezoito meses do bebê. Psico-USF, 12(1), 83-93.

Mezulis, A. H., Hyde, J. S., \& Clark, R. (2004). Father involvement moderates the effect of maternal depression during a child's infancy on child behavior problems in kindergarten. Journal of Family Psychology, 18(4), 575-588. doi:10.1037/0893-3200.18.4.575

Mian, L., Tango, L. A., Lopes, J., \& Loureiro, S. R. (2009). A depressão materna e o comportamento de crianças em idade escolar. Psicologia: Teoria e Pesquisa, 25(1), 29-37.

Mikelson, K. S. (2008). He said, she said: Comparing mother and father reports of father involvement. Journal of Marriage and Family, 70, 613-624. doi:10.1111/j.1741-3737.2008.00509.x

Monteiro, L., Veríssimo, M., Santos, A. J., \& Vaughn, B. E. (2008). Envolvimento paterno e organização dos comportamentos de base segura das crianças em famílias portuguesas. Análise Psicológica, 3(26), 395-409.

Organização Mundial da Saúde. (1997). CID-10: Classificação estatística internacional de doenças e problemas relacionados à saúde (10. ed. rev.). São Paulo, SP: Editora da Universidade de São Paulo.

Paulson, J. F., \& Bazemore, S. (2010). Prenatal and postpartum depression in fathers and its association with maternal depression. JAMA, 303(19), 1961-1969. doi:10.1001/jama.2010.605

Pizeta, F. A., Silva, T. B. F., Cartafina, M. I. B., \& Loureiro, S. R. (2013). Depressão materna e riscos para o comportamento e a saúde mental das crianças: Uma revisão. Estudos de Psicologia (Natal), 18(3), 429-437. doi:10.1590/S1413294X2013000300003
Pleck, J. H. (2010). Paternal involvement: Revised conceptualization and theoretical linkages with child outcomes. In M. E. Lamb (Ed.), The role of the father in child development ( $5^{\text {th }}$ ed., pp. 67-107). New York: Wiley.

Prado, A. B., Piovanotti, M. R. A., \& Vieira, M. L. (2007). Concepções de pais e mães sobre comportamento paterno real e ideal. Psicologia em Estudo, 12(1), 41-50. doi:10.1590/S141373722007000100006

Rienks, S. L., Wadsworth, M. E., Markman, H. J., Einhorn, L., \& Etter, E. M. (2011). Father involvement in urban low-income fathers: Baseline associations and changes resulting from preventive intervention. Family Relations, 60(2), 191204. doi:10.1111/j.1741-3729.2010.00642.x

Séjourné, N., Vaslot, V., Beaumé, M., Goutaudier, N., \& Chabrol, H. (2012). The impact of paternity leave and paternal involvement in child care on maternal postpartum depression. Journal of Reproductive and Infant Psychology, 30(2), 135-144. doi:10.1080/02646838.2012.693155

Silva, M. R., \& Piccinini, C. A. (2009). Paternidade no contexto da depressão pós-parto materna: Revisando a literatura. Estudos de Psicologia (Natal), 14(1), 5-12. doi:10.1590/ S1413-294X2009000100002

Smith, L. E., \& Howard, K. S. (2008). Continuity of paternal social support and depressive symptoms. Journal of Family Psychology, 22(5), 763773. doi:10.1037/a0013581

Souza, C. L. C., \& Benetti, S. P. C. (2008). Paternidade e desemprego: Características do envolvimento paterno e aspectos do relacionamento familiar. Contextos Clínicos, 1(2), 61-71.

Yoshida, A. (2012). Dads who do diapers: Factors affecting care of young children by fathers. Journal of Family Issues, 33(4), 451-477. doi:10.1177/0192513X11415358 